\title{
Estrogen-secreting adrenocortical tumor in a postmenopausal woman: a challenging diagnosis
}

\author{
L M Mermejo', F G Carvalho'1, C A F Molina², S Tucci², V F Muglia³, J Elias Jr33, P C L Elias', \\ M Castro ${ }^{1}$ and A C Moreira1 \\ 1Department of Internal Medicine, 2Department of Surgery and Anatomy, and ${ }^{3}$ Department of Radiology, \\ Hematology and Oncology, University of Sao Paulo Faculty of Medicine of Ribeirao Preto, Ribeirao Preto, São \\ Paulo, Brazil
}

\author{
Correspondence \\ should be addressed \\ to L M Mermejo \\ Email \\ Imermejo@hcrp.usp.br
}

\section{Summary}

Adrenocortical carcinoma (ACC) is a malignant disorder with rapid evolution and severe prognosis in adults and most produce cortisol and androgen. Estrogen-secreting adrenocortical carcinomas are extremely rare, especially in women, tend to be larger and have worse prognosis compared with other types of ACCs. We report the case of a 58-year-old woman who presented with bilateral breast enlargement and postmenopausal genital bleeding. She presented high estradiol ( $818 \mathrm{pg} / \mathrm{mL}-25$ times above upper normal limit for postmenopausal women) and testosterone (158 ng/dL - 2 times above upper normal limit) levels and no suppression of cortisol after overnight $1 \mathrm{mg}$ dexamethasone test (12.5 $\mu \mathrm{g} / \mathrm{dL}$; normal reference value: $<1.8 \mu \mathrm{g} / \mathrm{dL}$ ). The patient had no clinical features of cortisol excess. MRI showed a $12 \mathrm{~cm}$ tumor in the right adrenal. Clinical findings of bilateral breast enlargement and postmenopausal genital bleeding with no signs of hypercortisolism associated with hormonal findings of elevated estradiol and testosterone levels would indicate either an ovarian etiology or an adrenal etiology; however, in the context of plasma cortisol levels non-suppressive after dexamethasone test and the confirmation of an adrenal tumor by MRI, the diagnosis of an adrenal tumor with mixed hormonal secretion was made. The patient underwent an open right adrenalectomy and pathological examination revealed an ACC with a Weiss' score of 6 . Estradiol and testosterone levels decreased to normal range soon after surgery. She was put on mitotane treatment as adjuvant therapy, but due to side effects, we were unable to up-titrate the dose and she never achieved serum mitotane dosage above the desired $14 \mu \mathrm{g} / \mathrm{mL}$. The patient remained in good health without any local recurrence or metastasis until 5 years after surgery, when increased levels of estradiol $(81 \mathrm{pg} / \mathrm{mL}-2.5$ times above upper normal limit) and testosterone ( $170 \mathrm{ng} / \mathrm{dL}-2.1$ times above upper normal limit) were detected. MRI revealed a retroperitoneal nodule measuring $1.8 \times 1.2 \mathrm{~cm}$. The pathological finding confirmed the recurrence of the estrogensecreting ACC with a Weiss' score of 6 . After the second procedure, patient achieved normal estrogen and androgen serum levels and since then she has been followed for 3 years. The overall survival was 8 years after the diagnosis. In conclusion, although extremely rare, a diagnosis of an estrogen-secreting ACC should be considered as an etiology in postmenopausal women presenting with bilateral breast enlargement, genital bleeding and increased pure or prevailing estrogen secretion.

\section{Learning points:}

- Estrogen-secreting adrenocortical carcinomas are exceedingly rare in adults and account for $1-2 \%$ of adrenocortical carcinomas.

- Estrogen-secreting adrenal tumors can be present in females, but are even more rare, we found few cases described in the literature. In women, they present with precocious puberty or postmenopausal bleeding.

- Feminization in the context of an adrenal tumor is considered almost pathognomonic of malignancy. Feminizing ACCs tend to be larger and with worse prognosis compared with nonfeminizing ACCs. 


\section{Background}

Adrenocortical carcinoma (ACC) is a very rare tumor reported to occur in approximately 1-2 per million people per year (1). The prognosis is poor, with 5-year survival rates of approximately $30 \%$ in adults. Some patients present with advanced disease (stage IV), in which the 5-year survival is less than 5\% $(1,2)$. ACC can be divided into functional or nonfunctional tumors according to the hormone secretion status; however, most ACCs secrete cortisol and rarely secrete androgens and aldosterone (2).

Estrogen-secreting adrenal tumors are extremely uncommon; and some authors have used the term feminizing adrenal tumors (FATs) in men. However, it can be present also in females with precocious puberty or postmenopausal genital bleeding. In male, FATs can be present with signs of feminization such as gynecomastia, testicular atrophy, and/or diminished libido. Gynecomastia is the most common presenting complaint, with hypogonadism likely due to the suppression of gonadotrophins by the excess estrogens.

Feminization in the context of an adrenal tumor is considered almost pathognomonic of malignancy. Feminizing ACCs tend to be larger and with worse prognosis compared with nonfeminizing ACCs (3). In 1965, Gabrilove et al. (4) reported 20\% survival rate after 3 -year of the diagnosis.

FATs are mainly described in men with median age of 42 years (19-77 years) (5). The descriptions in women, to the best of our knowledge, are scanty. There are less than 10 women with estrogen secreting adrenocortical tumor described in the literature. Herein, we report the case of a postmenopausal woman with an estrogen-secreting ACC and her follow up after 8 years of the diagnosis.

\section{Case presentation}

A 58-year-old woman presented with bilateral breast enlargement and postmenopausal genital bleeding. After evaluation by a Gynecologist, the patient was submitted to a hysterectomy, with no improvement of breast enlargement. There was no history of exogenous steroids use.

\section{Investigation}

She was referred to hormonal assessment to an Endocrinologist, who observed high estradiol $(818 \mathrm{pg} / \mathrm{mL}$ - 25 times above upper normal limit for postmenopausal women) and testosterone (158 ng/dL - 2 times above upper normal limit) levels and no suppression of plasma cortisol after an overnight $1 \mathrm{mg}$ dexamethasone test $(12.5 \mu \mathrm{g} / \mathrm{dL}$; normal reference value: $<1.8 \mu \mathrm{g} / \mathrm{dL})$. The patient had no clinical features of cortisol excess. The gonadotropins levels were abnormally low (FSH; $0.3 \mathrm{mU} / \mathrm{mL}$; LH: $0.7 \mathrm{mU} / \mathrm{mL}$ ) for a menopause woman (Table 1). MRI showed a tumor measuring $12 \mathrm{~cm}$ in the right adrenal (Fig. 1A). Clinical findings of bilateral breast enlargement and postmenopausal genital bleeding with no signs of hypercortisolism associated with hormonal findings of elevated estradiol and testosterone levels would indicate either an ovarian etiology or an adrenal etiology; however, in the context of plasma cortisol levels non-suppressive after dexamethasone test and the confirmation of an adrenal tumor by magnetic resonance image, the diagnosis of an adrenal tumor with mixed hormonal secretion was performed.

\section{Treatment}

The patient underwent an open right adrenalectomy and pathological examination revealed an ACC with a Weiss' score of 6 . The immunohistochemistry was positive for inhibin $(3+/ 4+)$ and calretinin $(3+/ 4+)$. The final tumor classification was pT2NOM0. Molecular investigation for TP53 R337H and CTNNB1 mutations was negative (6).

After surgery, patient received additional adjuvant mitotane therapy as well as replacement therapy with prednisone and fludrocortisone for adrenal insufficiency during mitotane therapy. Three months after surgery, the bilateral breast enlargement improved and the estradiol level was $53 \mathrm{pg} / \mathrm{mL}$. However, due to gastrointestinal adverse effects of mitotane, the drug was withdrawn after 23 months and she never achieved serum mitotane dosage above the desired $14 \mu \mathrm{g} / \mathrm{mL}$.

Table 1 Biochemical profile at the diagnosis.

\begin{tabular}{|c|c|c|}
\hline Parameters & Values & Normal range \\
\hline Estradiol, pg/mL & 818 & $<32$ \\
\hline Testosterone, ng/dL & 158 & $20-80$ \\
\hline DHEAS, $\mu g / d L$ & 261 & $25-460$ \\
\hline 17-OHP, ng/dL & 250 & $<200$ \\
\hline Androstenedione, ng/dL & 142 & $60-250$ \\
\hline $\mathrm{LH}, \mathrm{mU} / \mathrm{mL}$ & 0.3 & $11-40$ \\
\hline $\mathrm{FSH}, \mathrm{mU} / \mathrm{mL}$ & 0.7 & $21-153$ \\
\hline Cortisol*, $\mu g / d L$ & 12.5 & $<1.8$ \\
\hline
\end{tabular}

*Cortisol after overnight $1 \mathrm{mg}$ dexamethasone suppression test.

17-OHP, 17- $\alpha$-OH progesterone; DHEA-S, DHEA sulfate. 

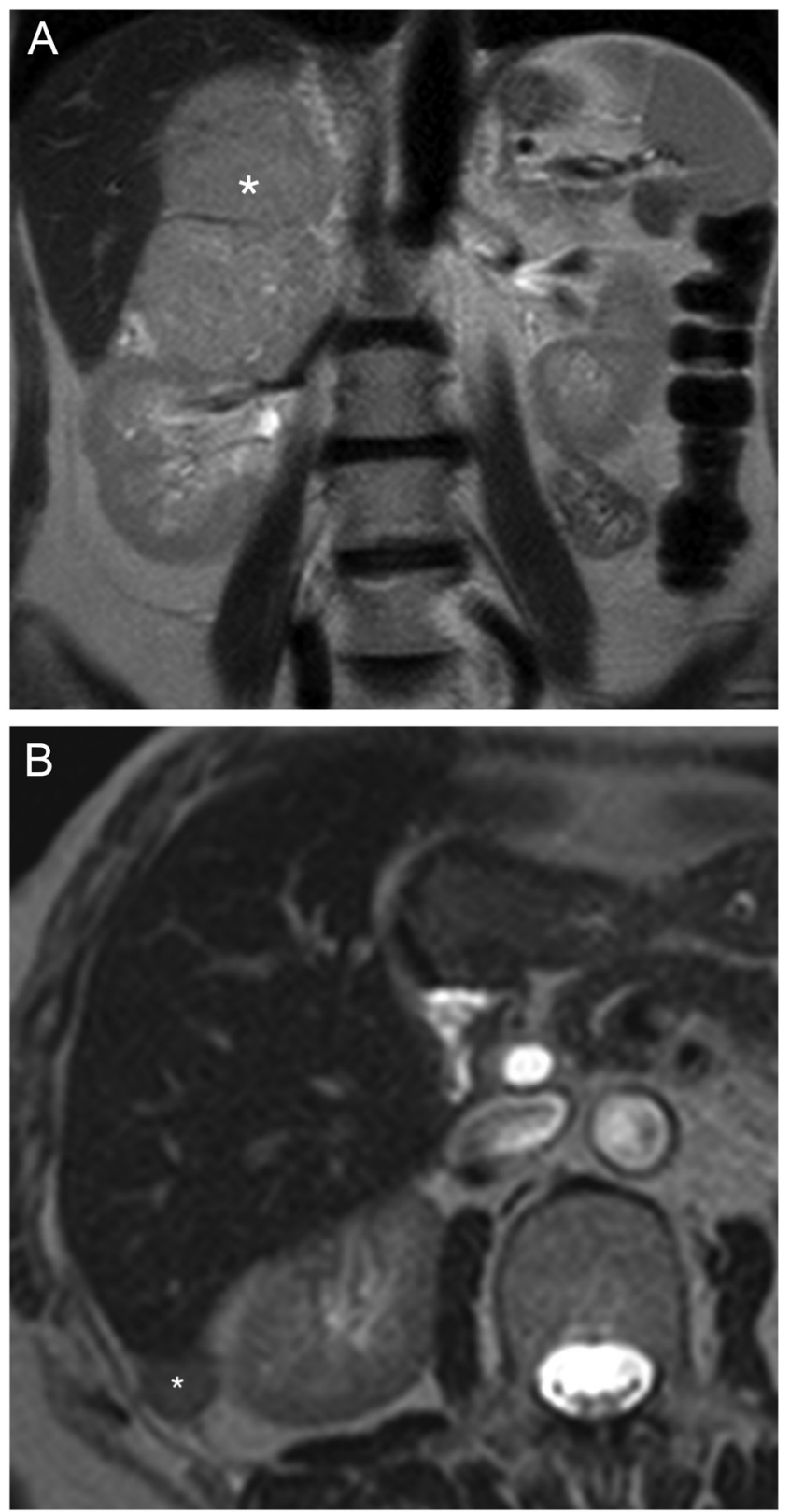

\section{Figure 1}

(A) Coronal T2-weighted MR image, at the clinical presentation showing a $12 \mathrm{~cm}$ intermediate-signal intensity tumor in the right adrenal gland (*). Pathological examination: ACC with a Weiss' score of 6 (Pt2n0m0). (B) Axial T2-weighted MR image 5 years after the first surgery revealed a right retroperitoneal nodule with the same imaging characteristics (asterisk).

\section{Outcome and follow-up}

The patient remained in good health without any local recurrence or metastasis until 5 years after treatment, when estradiol ( $81 \mathrm{pg} / \mathrm{mL}$ - 2.5 times above upper normal limit for postmenopausal women) and testosterone (170 ng/ $\mathrm{dL}-2.1$ times above upper normal limit) increased levels were detected (Table 2). MRI showed a retroperitoneal nodule measuring $1.8 \times 1.2 \mathrm{~cm}$ and patient was submitted to a second surgery (Fig. 1B). The pathological finding was a recurrence of the estrogen-secreting ACC with a Weiss' score of 6 . After the second procedure, estrogen and androgen serum levels normalized and since then she has been followed for 3 years. We tried to reuse mitotane but due to the side effects, it was not possible to keep the patient under mitotane treatment. During follow-up, we performed MRI annually. Thus, the overall survival of our patient has been, at least, 8 years since diagnosis.

\section{Discussion}

Estrogen-secreting adrenocortical carcinomas are exceedingly rare in adults and account for $1-2 \%$ of ACCs (3). They are prevalent in adult males, although they can be rarely observed in women and children. Here we describe a 58-year-old woman with bilateral breast enlargement and postmenopausal genital bleeding who was submitted to a hysterectomy after evaluation by a gynecologist, with no improvement in breast enlargement. After the endocrinologist's evaluation, high estradiol and testosterone levels were observed with no suppression of plasma cortisol after an overnight $1 \mathrm{mg}$ dexamethasone test.

We believe that estrogen hypersecretion by the tumor is the main cause of the signs of bilateral breast enlargement and postmenopausal genital bleeding found in our patient. However, elevated levels of estrone and estradiol have also been found in virilizing tumors without estrogens co-secretion. Indeed, estrogen excess can result from augmentation in aromatase activity within the tumor leading to an increased synthesis imbalance between high estrogen and low free androgens as suggested by

Table 2 Hormonal follow-up.
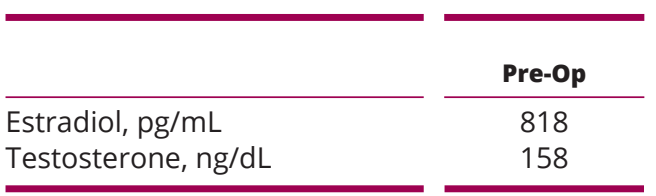

\begin{tabular}{|c|c|c|}
\hline \multicolumn{3}{|c|}{ Post-Op first surgery } \\
\hline 5 days & 1 year & 5 years \\
\hline 67 & 59 & 81 \\
\hline 20 & $<10$ & 170 \\
\hline
\end{tabular}

\begin{tabular}{|c|c|c|}
\hline \multicolumn{3}{|c|}{ Post-Op second surgery } \\
\hline 5 days & 1 year & 5 years \\
\hline 75 & 67 & 12 \\
\hline$<10$ & $<10$ & $<10$ \\
\hline
\end{tabular}

Op, operation. 
Zayed et al. (7). These authors studying a woman with ACC and high serum levels of androgens and estrone estimated that half of the circulating estrone had come directly from the tumor and half from peripheral conversion of androstenedione. Our patient clearly had evidence of E2 production and secretion by the tumor itself, given the very high $\mathrm{E} 2$ and moderately elevated serum testosterone. Recently, Wu et al. showed high expression of CYP19A1 as well as a high expression of beta-catenin nuclear staining in four feminizing ACC tumors and in cell lines (8).

As these tumors can also secrete cortisol with or without clinical symptoms of Cushing's syndrome (9), it is mandatory to evaluate cortisol levels after overnight $1 \mathrm{mg}$ dexamethasone suppression test. Indeed, besides hyperestrogenism, our patient presented a non-suppressible cortisol levels after dexamethasone suppression test and high levels of androgens suggesting a mixed hormonal secretion.

In 1965, the first literature review recorded 52 FATs, all of these patients were men, presenting gynecomastia (98\%), a palpable abdominal mass (58\%), and testicular atrophy $(52 \%)$. Patients with FATs usually complain of diminished libido (48\%) and breast tenderness (42\%) (4). Estrogen secreting ACCs are exceptionally rare in women, accounting for less than 10 cases; there is no clear reason for gender difference, however since signals and symptoms are more prominent in male, women can be underdiagnosed.

FATs have a very poor prognosis. Mortality among adult males is high and survival rate is less than $20 \%$ after 3 years of tumor resection $(3,4,10)$. Common sites of intraabdominal metastases include liver, intestine, peritoneum, and retroperitoneal lymph nodes. Extra-abdominal metastases may be observed in lungs, pleura, bones, and other sites. Treatment is based mainly on surgery with complete resection of the tumor and metastases, when possible. Medical therapy with mitotane ( $\left.\mathrm{O}^{\prime} \mathrm{p}^{\prime} \mathrm{DDD}\right)$ can be used as both adjuvant treatment or primarily in advanced disease. For recurrent or metastatic FATs, radiation therapy and various chemotherapies can be considered. However, with the exception of mitotane, there is no other effective medication. Aromatase inhibitors have been used without success (11)

Remarkably, in the present case reported, after 5 years of the first surgery, the patient presented a local recurrence of the tumor and a second surgical procedure was performed. Of note, she has used adjuvant mitotane therapy for only 23 months as she did not tolerate mitotane therapy side effects. After the second procedure, patient has achieved normal estrogen and androgen serum levels and has been followed for the last 3 years. Molecular evaluation of TP53 $\mathrm{R} 337 \mathrm{H}$ and CTNNB1 mutations was negative. We have previously demonstrated that TP53 p.R337H and CTNNB1 mutations correlated with poor prognosis in adults and children with adrenocortical tumors, respectively (6). In addition, data from the Cancer Genome Atlas show a higher rate of Wnt-related mutations in functional ACCs compared with nonfunctional ACCs $(48.9 \%$ vs 23.1\%) (12).

In conclusion, we presented a female patient with estrogen-secreting adrenocortical carcinoma, who has been treated by two surgeries and temporary mitotane adjuvant therapy, but most important she has been monitored through continuous follow-up visits. Although extremely rare, a diagnosis of an estrogen-secreting ACC should be considered as an etiology in postmenopausal women presenting with bilateral breast enlargement, genital bleeding and increased, pure or prevailing, estrogen secretion.

\section{Declaration of interest}

A C Moreira is a Senior Editor of Endocrinology, Diabetes and Metabolism Case Reports. A C Moreira was not involved in the review or editorial process for this paper, on which he is listed as an author.

\section{Funding}

This research did not receive any specific grant from any funding agency of commercial or no-for-profit sector.

\section{Patient consent}

The patient has submitted written informed consent to publish this observation.

\section{Author contribution statement}

All of the authors made a major contribution to the patient's clinical care and the intellectual content of this case report.

\section{References}

1 Fassnacht M, Kroiss M \& Allolio B. Update in adrenocortical carcinoma. Journal of Clinical Endocrinology and Metabolism 201398 4551-4564. (https://doi.org/10.1210/jc.2013-3020)

2 Else T, Kim AC, Sabolch A, Raymond VM, Kandathil A, Caoili EM, Jolly S, Miller BS, Giordano TJ \& Hammer GD. Adrenocortical carcinoma. Endocrine Reviews 201435 282-326. (https://doi. org/10.1210/er.2013-1029)

3 Moreno S, Guillermo M, Decoulx M, Dewailly D, Bresson R \& Proye Ch. Feminizing adreno-cortical carcinomas in male adults. A dire prognosis. Three cases in a series of 801 adrenalectomies and 
review of the literature. Annales d'Endocrinologie $2006 \mathbf{6 7} 32-38$. (https://doi.org/10.1016/s0003-4266(06)72537-9)

4 Gabrilove JL, Sharma DC, Wotiz HH \& Dorfman RI. Feminizing adrenocortical tumors in the male. A review of 52 cases including a case report. Medicine 196544 37-79.

5 Chentli F, Bekkaye I \& Azzoug S. Feminizing adrenocortical tumors: literature review. Indian Journal of Endocrinology and Metabolism 2015 19 332-339. (https://doi.org/10.4103/2230-8210.152764)

6 Mermejo LM, Leal LF, Colli LM, Fragoso MC, Latronico AC, Tone LG, Scrideli CA, Tucci S, Martinelli CE, Yunes JA, et al. Altered expression of noncanonical Wnt pathway genes in paediatric and adult adrenocortical tumours. Clinical Endocrinology 201481 503-510. (https://doi.org/10.1111/cen.12462)

7 Zayed A, Stock JL, Liepman MK, Wollin M \& Longcope C. Feminization as a result of both peripheral conversion of androgens and direct estrogen production from an adrenocortical carcinoma. Journal of Endocrinological Investigation 199417 275-278. (https://doi. org/10.1007/BF03348975)

8 Wu L, Xie J, Jiang L, Su T, Ye L, Zhou W, Jiang Y, Zhang C, Ning G \& Wang W. Feminizing adrenocortical carcinoma: the source of estrogen production and the role of adrenal-gonadal dedifferentiation. Journal of Clinical Endocrinology and Metabolism 2018103 3706-3713. (https://doi.org/10.1210/jc.2018-00689)

9 Fukai N, Hirono Y, Yoshimoto T, Doi M, Ohtsuka Y, Homma K, Shibata H, Sasano H \& Hirata Y. A case of estrogen-secreting adrenocortical carcinoma with subclinical Cushing's syndrome. Endocrine Journal 200653 237-245. (https://doi.org/10.1507/ endocri.53.237)

10 Bhettay E \& Bonnici F. Pure oestrogen-secreting feminizing adrenocortical adenoma. Archives of Disease in Childhood $1977 \mathbf{5 2}$ 241-243. (https://doi.org/10.1136/adc.52.3.241)

11 Kidd MT, Karlin NJ \& Cook CB. Feminizing adrenal neoplasms: case presentations and review of the literature. Journal of Clinical Oncology 201129 e127-e130. (https://doi.org/10.1200/ JCO.2010.31.4799)

12 Zheng S, Cherniack AD, Dewal N, Moffitt RA, Danilova L, Murray BA, Lerario AM, Else T, Knijnenburg TA, Ciriello G, et al. Comprehensive pan-genomic characterization of adrenocortical carcinoma. Cancer Cell 201630 363. (https://doi.org/10.1016/j. ccell.2016.07.013)

Received in final form 18 January 2021

Accepted 18 February 2021 